\title{
Energy Aware Algorithm for Clustering in Wireless Network
}

\author{
Pradipta Ghosh \\ Department of Electronics and Telecommunication \\ Engg, Jadavpur University, \\ Kolkata-700032 \\ Nikita Pandey \\ Department of Information Technology, \\ Narula Institute of Technology, \\ Kolkata-700109
}

\author{
Sayani Nandy \\ Department of Information Technology, Narula \\ Institute of Technology, \\ Kolkata-700109
}

\begin{abstract}
In numerous applications, self-organizing property of Wireless Sensor Networks (WSN) is an important characteristic. It calls for decompositions of the network into clusters of desirable bound. Cluster based WSN can enhance the whole networks lifetime. In every cluster, the cluster head $(\mathrm{CH})$ plays an important role in aggregating and forwarding data sensed by other non-leader nodes. A major issue in the cluster based approach in WSN is the selection of proper cluster head and attainment of desirable cluster size by maximum number of clusters formed, keeping into consideration the inherent constraints such as limited battery energy, failure of nodes, selfish behavior of nodes, limited bandwidth etc, which inhibits superior message efficiency. This research paper presents a clustering approach termed as Sequential Multi-Clustering Protocol (SMCP) incorporating node deployment, which enhances the lifetime of the network. This Protocol is applied on some popular clustering algorithms like 'Expanding Ring', 'Rapid' and 'Persistent' along with our own clustering algorithm 'Message Based Memory Efficient Clustering Algorithm' (MMEC) to cluster an entire topology of the network. Simulation results mainly in MATLAB interpreter shows the effectiveness of clustering using SMCP protocol.
\end{abstract}

Keywords: Clustering, Sensor Networking, Network Protocol.

\section{INTRODUCTION}

Recent advancement in technology made the manufacturing of small and low cost sensors technically and economically feasible, which are composed of a single chip with embedded memory, processor, and transceiver. In general such sensors are also equipped with data processing and communication capabilities.

The technological advances in micro-electro-mechanical systems and low power and highly integrated digital electronics have led to the development of micro-sensors [1-5]. The sensors measure ambient condition in environment surrounding it and then transform them into an electric signal. Processing such a signal reveals important properties about objects located and/or events happening in the vicinity of that particular sensor. A large number of these disposable sensors can be grouped or networked in many applications that require unattended operations.

The key feature of a typical sensor network is that their nodes are un-tethered and unattended. These sensors possess the ability to communicate either among each other or directly to an external base-station (BS). No doubt a greater number of sensors allows for sensing over a large geographical regions with greater accuracy than small number of sensors. WSN finds its applications in diverse fields. In the field of healthcare, sensor nodes have been deployed to monitor the condition of patients [6]. Sensor nodes have also found its application in cattle ranch monitoring [7], cattle control [8], zebra herd monitoring [9], etc. Efficient flood forecasting systems utilizing WSN, are also being developed $[10,11]$ nowadays. With the flourishing growth in the use of WSN, the size and complexity of the networks will increase. The sensor nodes are essentially low-cost, low-power devices capable of communicating over short distances and processing some data. These nodes are generally densely deployed in the concerned region either in a regular or irregular manner. As WSN could get exposed to adverse environments [12] e.g. - disaster monitoring, they must have a self organizing capability and immunity to node failures. So it is expected that a large number of cheap, simple sensor devices will be randomly scattered over the region of interest. A network, deployed with large number of sensor nodes remains functional [13] when all nodes or a predefined percentage of nodes are alive in a network. Network lifetime [14] thus becomes an important parameter for efficient design of sensor networks. In case of WSNs, the lifetime can be taken as the time from inception of the nodes to the time when the network becomes non-functional. A network may become non-functional when a single node dies or when a particular percentage of nodes die depending on requirement. Each node is provided with transmit power control and Omni directional antenna and therefore can vary the areas of its coverage [15]. Each node is battery powered and has limited processing and memory capabilities. Therefore it is critical that these resources are to be used in a judicious manner in order to maximize the benefit from the network before it dies. Although there is a cooperative effort from the device research community towards designing low power hardware and efficient energy sources, the network research community has also realized that inefficient algorithms at the various networking layers can result in nodes dying prematurely. There are several proposals at the MAC ([16, 17]) and Network layers ([18, 19]). Thus it becomes necessary to prevent single points of failure which in turn calls for distributed algorithms. The self-organizing property of WSN requires network decomposition into clusters of specific sizes. The main aim should be to minimize the message complexity of the clustering algorithms and to ensure the cluster formed attain 
the specific bound. Lack of sufficient power in the nodes and limited bandwidth of the wireless medium [6] makes the task of clustering more challenging.

Clustering is defined as the grouping of similar objects or the process of finding a natural association among some specific objects or data that finds applications in many fields. Clustering, specifically in sensor networks, could be used to solve a verity of problems. For example, [20] uses clusters to transmit processed data to base stations, hence minimizing the number of nodes that take part in long distance communication which directly affect the overall system energy dissipation. Apart from sensor networks, clustering has been applied tremendously and successfully in fields like VLSI-CAD and data mining [21]. A classical analytic VLSI placer [22] uses clustering for efficient standard cell placement.

The very first clustering algorithm was the expanding ring algorithm [22]. However the no. of messages used by the algorithm in case of dense topologies is too large. Rajesh Krishnan and David Storobinski [23] suggested messageefficient algorithms, based on allocation of local growth budget to neighbors. The two algorithms suggested are the Rapid and Persistent approach. Both are far more efficient in terms of message complexity than the expanding ring approach. The Rapid algorithm generally uses fewer messages than the persistent approach but very often fails to achieve the specified cluster size. The second algorithm is an improved version of the Rapid approach as it persistently tries to produce a cluster of the specific size in exchange of some extra messages.

In this paper, we will also use an algorithm developed by our research group namely 'Memory based Message Efficient Clustering' (MMEC) algorithm [24] incorporating a probabilistic approach which predetermines the number of nodes to be included in the next hop. The algorithm was developed in the basis of achieving a pinnacle in the lifetime of the network by maintaining its self organization property. It focuses mainly in reducing message complexity. This paper mainly concentrates on protocol for multi-clustering abbreviated as SMCP. This protocol mainly focuses upon reduction of message complexity and to maintain the number of cluster within a range along with no of elements in each cluster within desired value while clustering the entire WSN. The effectiveness of this method is justified by a set of simulations using four clustering algorithms and the results are presented. The latest network programming tool - NS 2.33 has been used in evaluating the performance of the proposed algorithm along with MATLAB 7.8. The rest of the paper includes previous works in literature in section 2 , our proposed clustering algorithm SMCP in section 3, simulation based observation in section 4 followed by a brief conclusion in section 5 .

\section{PREVIOUS WORKS}

Some of present clustering algorithms like LCA [25], RCC [26] and CLUBS [27], have $\mathrm{O}(\mathrm{n})$ convergence time, where $\mathrm{n}$ is the number of sensor nodes in the network. Baker et. al. proposed Linked cluster algorithm (LCA) [25, 26] which is among the early ones on clustering of wireless networks. The primary focus is to form an efficient network topology that can handle the mobility of nodes. In [29], Lin et. al. proposed another clustering algorithm termed as 'adaptive clustering'. They employed an IDbased cluster selection scheme similar to [25] which established a single-hop intra-cluster topology similar to LCA. In [30], another clustering method termed as random competition based clustering (RCC) is designed for mobile ad hoc networks, also applicable to WSN. The main focus of RCC is cluster stability in order to support mobile nodes. The RCC algorithm applies the First Declaration Wins rule, in which any node can "govern" the rest of the nodes in its radio coverage if it is the first to claim being a $\mathrm{CH}$. In [27], Nagpal and Coore proposed CLUBS, an algorithm that forms clusters through local broadcast and converge in a time proportional to the local density of nodes. Unlike most of the published schemes, the goal of Banerjee and Khuller is to form a multi-tier hierarchical clustering [31]. Their method is termed as Hierarchical control clustering. In grouping of nodes, several cluster properties such as cluster size and the degree of overlap that are useful for the management and scalability of the hierarchy, are also considered. In this scheme, any node in the WSN can initiate the cluster formation process. Initiator with least node ID will take precedence, if multiple nodes started cluster formation process at the same time. Zhang et. al. proposed another clustering algorithm in [32], called GS3, to achieve self-configuration of a wireless network into a cellular hexagonal structure. According to the authors, ignoring the geographical boundary of clusters can be unwise, especially or very large network. So, they defined the radius of the circle that contains all nodes in the cluster as a measure for the geometrical size. Bandyopadhyay and Coyle [33] proposed EEHC (Energy Efficient Hierarchical Clustering), a distributed, randomized clustering algorithm for WSN with the objective of maximizing the network lifetime. CHs collected the sensors' readings in their individual clusters and send an aggregated report to the basestation. Their technique is based on two stages; initial and extended.

Clustering algorithms that converge completely in a fixed number of iterations, regardless of the size of the nodes population, are called constant convergence time clustering algorithms. Among these algorithms, Low Energy Adaptive Clustering Hierarchy (LEACH) is one of the most popular clustering algorithms for WSN [34]. It forms clusters based on the received signal strength and uses the $\mathrm{CH}$ nodes as routers to the base-station. All the data processing such as data fusion and aggregation are local to the cluster. LEACH forms clusters by using a distributed algorithm, where nodes make autonomous decisions without any centralized control. Fast Local Clustering service (FLOC) [35] is a distributed technique that produces approximately equal size clusters with minimum over-lap. The assumed radio model classifies nodes based on their proximity to the $\mathrm{CH}$ into inner (I-band) and outer (O-band). Unlike other distributed clustering schemes, ACE (Algorithm for Cluster Establishment) employs an emergent algorithm [36]. Emergent algorithms much like artificial neural networks evolve to optimal solution through a mix of local optimization steps. Hybrid Energy-Efficient Distributed Clustering (HEED) [37] is a distributed clustering scheme in which $\mathrm{CH}$ nodes are picked from the deployed sensors. HEED considers a hybrid of energy and communication cost when selecting CHs. Unlike LEACH, it does not select cell-head nodes randomly. Ding et al. [38] have proposed Distributed Weight-Based Energy-Efficient Hierarchical Clustering (DWEHC) to achieve more aggressive goals compared to HEED. DWEHC proceeds in a distributed manner and has $\mathrm{O}(1)$ time complexity. Both DWEHC and HEED are similar in many ways; every node in the network participates in the clustering process, they do not make any assumption about the network size and consider energy reserve in $\mathrm{CH}$ selection. However, Youssef et al. [39] argued that to guarantee some degree of overlap among clusters can facilitate many applications like inter-cluster routing, topology discovery 
and node localization and recovery from cluster head failure etc. They proposed MOCA, a randomized, distributed Multi-hop Overlapping Clustering Algorithm for organizing the sensors into overlapping clusters. The goal of the clustering process is to ensure that each node is either a $\mathrm{CH}$ or within $\mathrm{k}$ hops from at least one $\mathrm{CH}$, where $\mathrm{k}$ is a preset cluster radius. Wang et al. [40] promoted the idea of clustering the WSN based on the queries and attributes of the data and proposed a new algorithm, Attribute-based clustering. The main motive is to achieve efficient dissemination of data in the network. The concept resembles the data-centric design model of WSN. In [41], Li et al. introduced a new clustering algorithm called EEUC to balance the energy consumption well among all sensor nodes and achieves an obvious improvement on the network lifetime. There are many other algorithms in various literatures [42-47].

\section{PROPOSED WORK}

\subsection{Sequential Multi clustering Protocol (SMCP)}

In this section, the proposed sequential multi-clustering protocol is described. Since this is a sequential clustering method only one cluster can be generated at a moment. That means until the previous cluster formation is complete next cluster cannot be generated. The most important thing of clustering is proper selection of cluster heads which is the primary focus of any clustering method. We must also keep in mind the available energy, message complexity and most of the important parameters regarding any WSN. Our proposed method can be divided into few subparts. In the entire method we assumed that the Base Station has a complete knowledge of all the nodes of the network and also it can communicate any node when necessary (this may be direct communication or may be indirect communication by routing protocol). Also we assume that the BS station determines the cluster head according to the algorithm and then send necessary information to the cluster heads. Also the BS updates its information after formation of each cluster by communicating through the cluster head.

\subsection{Selection of Initial Cluster Head}

In any sequential clustering algorithm, the selection of the first cluster head is the most crucial part. Only a proper selection of initial cluster head leads towards splendid success of the entire clustering process. For example in Figure 1 if any corner element is selected as cluster head, then the initial cluster will contain many hops. Also this cluster will have less number of neighboring sensor nodes; this may hamper the overall process. So in our proposed method, the first cluster head is not selected randomly from the entire set of available nodes. Let us assume that BS knows the entire topology of the network and it can command any sensor node to start acting like a cluster head. The entire paper is based on that assumption. This is also valid for a network without any assumption but the results will be slightly different. In our algorithm the first cluster head is selected as the center of the densest region of the entire topology. The density is determined by considering a circle of certain area and calculating the number of nodes within it. Again, as time complexity of this process is much large we need some procedure to reduce it. We can reduce the total area of this process by taking the average and the standard deviation of the co-ordinates of the nodes.

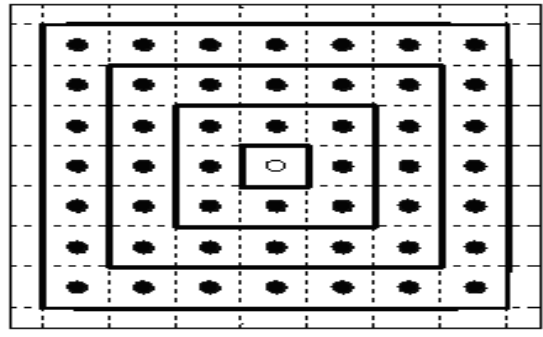

Figure 1. Ring formation containing the number of nodes included in each hop of expanded ring algorithm in the densest topology criterion

Let $\left(x_{i}, y_{i}\right)$ be the location of $\mathrm{i}^{\text {th }}$ node in the topology. Now let co-ordinates of the mean point (M.P) are $\left(x_{m}, y_{m}\right)$. Then, $x_{m}=\left(\sum_{i} x_{i}\right) / N \quad y_{m}=\left(\sum_{i} y_{i}\right) / N$

Now standard deviations are, $x_{s d}=\sqrt{\left(\sum_{i}\left(x_{m}-x_{i}\right)^{2} / N\right)}$

$y_{s d}=\sqrt{\left(\sum_{i}\left(y_{m}-y_{i}\right)^{2} / N\right)}$ and

Then we only examine the area within plus-minus one standard deviation along any co-ordinate axis around the M.P (i.e. $\left(x_{m}-x_{s d}\right) \leq x \leq\left(x_{m}+x_{s d}\right)$

and

$\left(y_{m}-y_{s d}\right) \leq y \leq\left(y_{m}+y_{s d}\right)$. Now the centers of the circles are chosen randomly within the determined area. We observed that taking the M.P as centre gives the highest density in some cases. We can also use some optimization algorithm to determine the starting cluster head. In Figure 2, the darkened node (or dot) is the M.P and the dashed area is the area selected for initial cluster head searching.

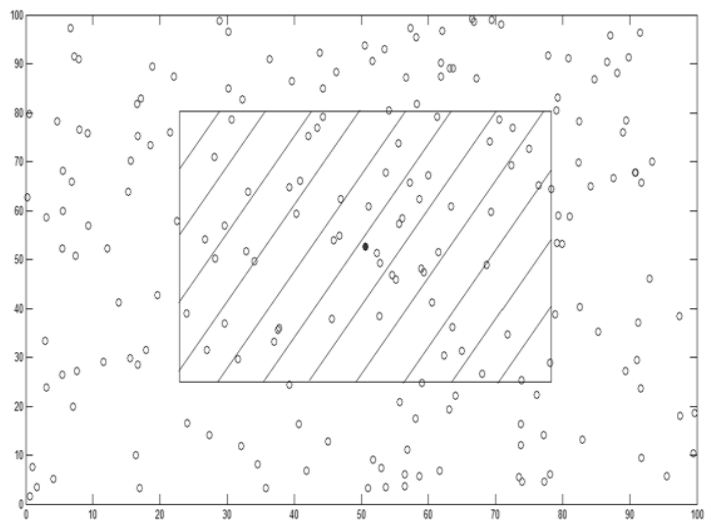

Figure 2. Representation of initial cluster head selection area 


\subsection{Clustering}

After the selection of initial cluster, the first cluster formation is initiated. After the cluster formation is complete, the last hop of the formed cluster marks their available (i.e. un-clustered) neighbor nodes so that they cannot be selected as cluster head. It can be inferred that all the nodes that are already clustered cannot be selected as cluster heads either. So they are marked as well. After the first cluster is formed, we have to select next cluster head.

\subsection{Selection of Next Cluster Head}

Now there are two types of selection procedure is offered in our algorithm for selecting the next cluster head.
Case 1: Previously we have provided a method of marking the neighbor nodes of previously clustered nodes. It is stated that the un-clustered neighborhood nodes of the respective last hops of the clusters, that are formed already, are marked. Now one of these nodes, that are marked for not to be selected as cluster head, is randomly chosen. The chosen node checks its immediate neighborhood for nodes available and unmarked (i.e. Unclustered and eligible of being selected as a cluster head) for selection of next cluster head. If there is a number of nodes are available, then any one of them can be selected as cluster head. So we have adopted a randomized approach toward selection of cluster head i.e. one of the sorted out nodes is selected randomly. This is shown in Figure 3.

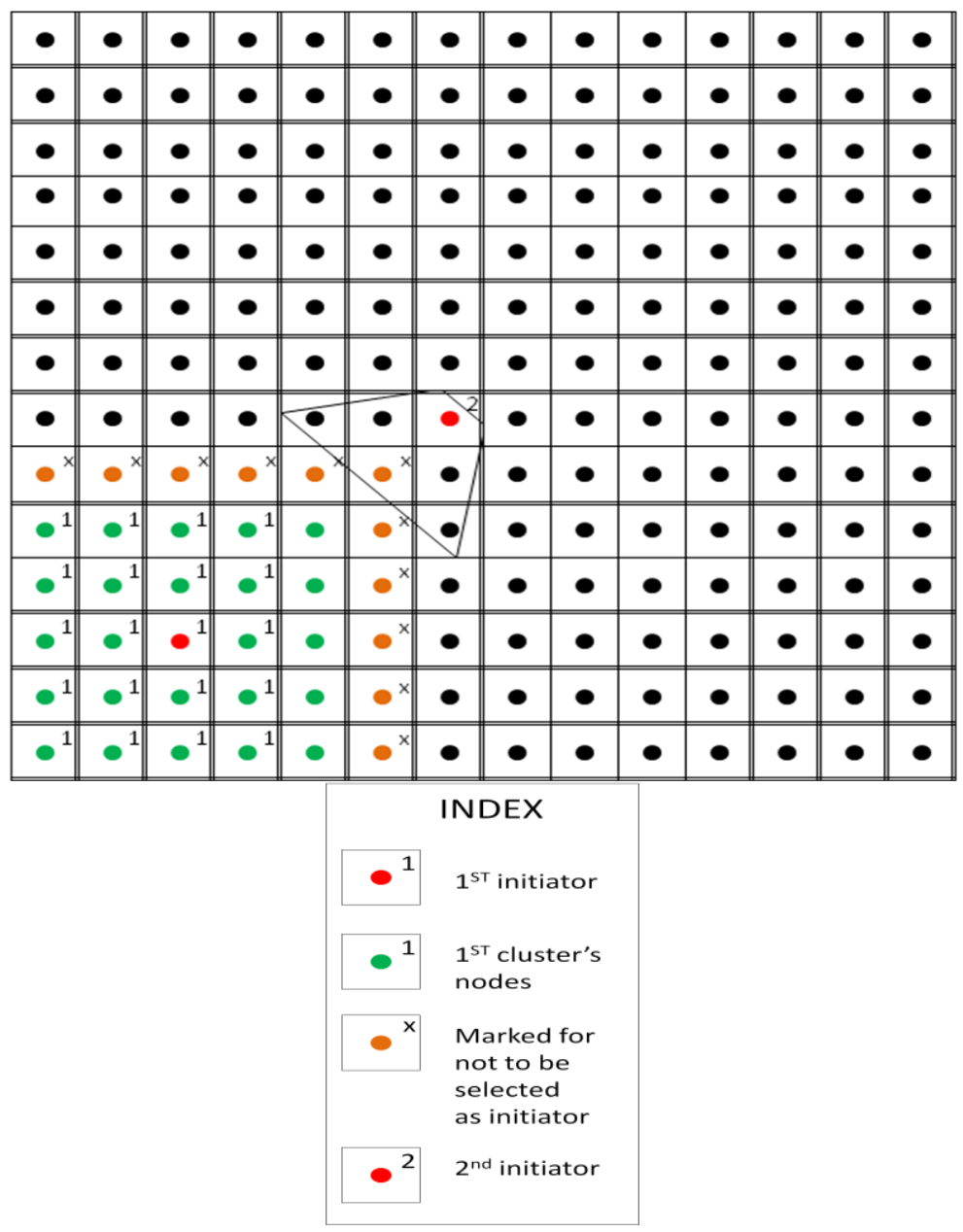

Figure 3. Representation of cluster head selection protocol for case 1

Case 2: Beside the first method of selection, another approach towards head section is given equal priority. This approach mainly focuses upon reduction of the message count in course of selection of cluster heads. We have a set of nodes that cannot be selected as cluster head. So we can easily select any one node among the rests (i.e. nodes not included in that set) as cluster head. This approach is also randomized. Any one of the nodes, available for selection as cluster head, is selected randomly as the next cluster head. This is shown in Figure 4.

These two types of selection method are given probability of 0.5 . The probability can be set to any value within 0.3 to 0.7 . We have chosen 0.5 to provide both operations equal occurring probability since these methods don't perform well alone. If both of these methods fail, then we check for nos. of un-clustered and unmarked nodes. If the number is high enough our algorithm will randomly select one of these left-out nodes as cluster head in the high density region.

Immediately after selection of each cluster head, the clustering is performed. The clustering is performed using the available clustering algorithms like expanding ring, rapid etc. The message exchange schedule of such clustering is same as the clustering algorithms used. After the formation of next cluster, previously describes steps are repeated again and again until most of the nodes are clustered. Again, we assume that the Base Station has a count of the no of clustered nodes. 


\subsection{Finalization}

It is observed that by using our method 1-3 nodes are left out after the clustering is complete. So we have included another mechanism to include them also. They are included to their nearest cluster at the expenses of one or two extra overhead.

Our model in algorithm form is given below with assumption that every node has a unique node ID and every node knows it neighbor:

Deploy Nodes;

Determine M.P $\left(x_{m}, y_{m}\right)$;

Determine $x_{s d \text { and }} y_{s d}$;

Draw the region of selection of initial Cluster Head;

For $\mathrm{i}=1: 50$

Randomly choose a point to determine the density around it;

Determine the density as stated before;

End For;
Determine the highest value of density and corresponding center point;

Choose the closest node to the center point as cluster head;

Form the 1st cluster;

While 1

Determine Next Cluster Head according to the rules stated above;

Perform clustering;

If No of unmarked nodes $=0$

Break;

End If;

End While;

Determine the No of Un-clustered Nodes;

Include the Nodes to their nearest cluster;

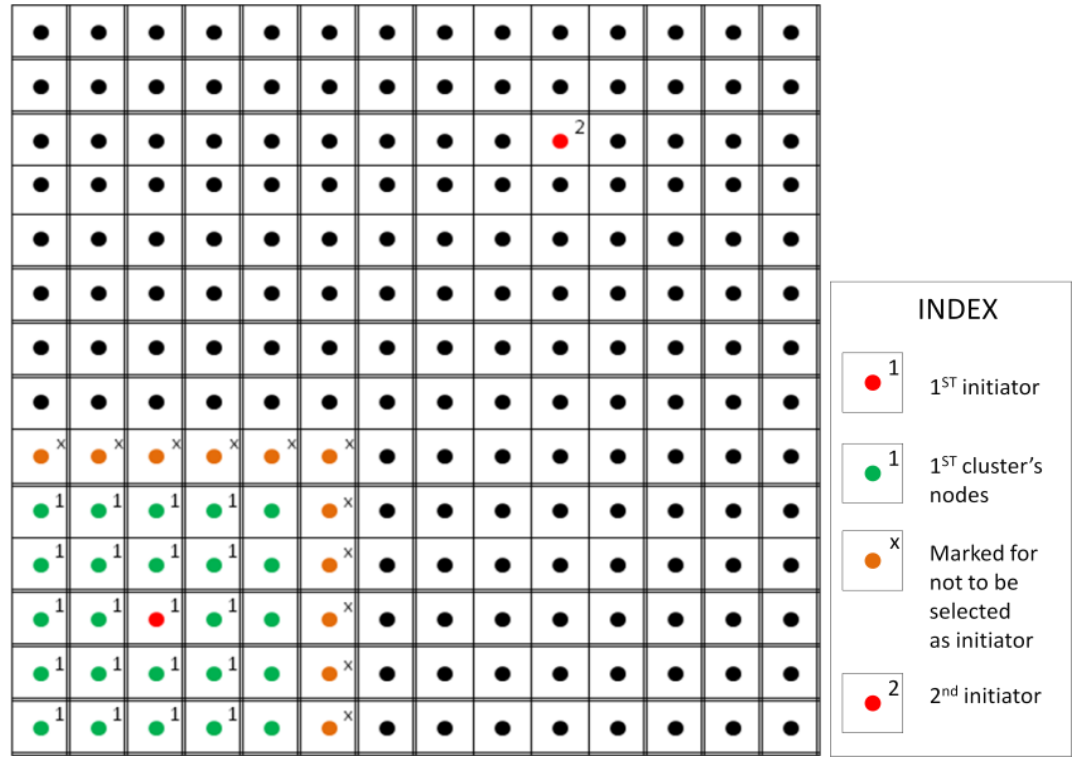

Figure 4. Representation of cluster head selection protocol for case 2

\section{OBSERVATIONS}

For checking the applicability of our SMCP method, at first we have created the WSN topology over which all the clustering algorithms Expanded Ring, Rapid, Persistent and MMEC [22, $23,24]$ run in NS 2.33. The average outcomes of the simulation are given here for two different topologies.

\section{Topology 1}

In the table 1 , we presented the results over the topology 1 with the corresponding parameters as follows.

Length of the square 2-D area over which the network is created in meter $=100$
Total number of nodes in the network $=500$

The range of the nodes in meter $=7.5$

Max no of nodes in a the cluster $=25$

So, No of Desired Cluster $=20$

Topology 2

In the table 2, we presented the results over the topology 2 with the corresponding parameters as follows.

Length of the square 2-D area over which the network is created in meter $=200$

Total number of nodes in the network $=1000$

The range of the nodes in meter $=7.5$

Max no of nodes in a the cluster $=25$

So, No of Desired Cluster $=40$ 
From Table 1 and Table 2 we can see that our proposed multicluster algorithm performs well for all the clustering algorithms. We can also see that the no. of clusters for each of the algorithms is as per our assumptions. Number of clusters is the highest for rapid and for rest of the algorithms it is almost same. The message complexity of our method is same as of the used clustering algorithm i.e. expanding ring, rapid, persistent or MMEC respectively. From the tabulated results we can firmly establish this fact. No of message is highest for expanding ring algorithm compared to other algorithms. But it is also true that the no of message exchanged is only the number of those used by any clustering algorithm. In our method some extra message is needed to mark the adjacent sensor nodes for the selection of next cluster head. This value depends on the number of cluster formed and its neighboring un-clustered network. In worst case phenomenon using deployment protocol, if the average no of hops in each cluster is ' $m$ ' and no of clusters is ' $n$ ' then the extra message needed is $\left(8^{*} \mathrm{~m}^{*} \mathrm{n}\right)$. But practically this value is very small compared to the worst case phenomenon. Only for the first few cluster formation all the neighbor nodes are unmarked and un-clustered. As the nos. of formed clusters increases, the no. of such nodes to be marked decreases rapidly. We can also see that the average no of left-out nodes is very small i.e. within 5 or both the cases. These results are obtained from simulations based on NS 2.33. The average values are taken over 25 runs. Figure 5 shows the number of elements in each cluster for all four clustering algorithms.

Table 1. Results of simulation over topology 1

\begin{tabular}{|c|c|c|c|c|}
\hline $\begin{array}{c}\text { TOPOLOGY } \\
\mathbf{1}\end{array}$ & $\begin{array}{c}\text { Expanding } \\
\text { Ring }\end{array}$ & Rapid & Persistent & MMEC \\
\hline $\begin{array}{c}\text { No of Clusters } \\
\text { No. Messages } \\
\text { exchanged }\end{array}$ & 22 & 29 & 22 & 21 \\
\hline $\begin{array}{c}\text { Avg. No. of un- } \\
\text { clustered nodes }\end{array}$ & 2 & 886 & 558 & 476 \\
\hline
\end{tabular}

Table 2. Results of simulation over topology 2

\begin{tabular}{|c|c|c|c|c|}
\hline $\begin{array}{c}\text { TOPOLOGY } \\
\text { 2 }\end{array}$ & $\begin{array}{c}\text { Expanding } \\
\text { Ring }\end{array}$ & Rapid & Persistent & MMEC \\
\hline No of Clusters & 44 & 55 & 43 & 43 \\
\hline $\begin{array}{c}\text { No. Messages } \\
\text { exchanged }\end{array}$ & 1620 & 1543 & 1146 & 1122 \\
\hline $\begin{array}{c}\text { Avg. No. of un- } \\
\text { clustered nodes }\end{array}$ & 2 & 5 & 2 & 1 \\
\hline
\end{tabular}




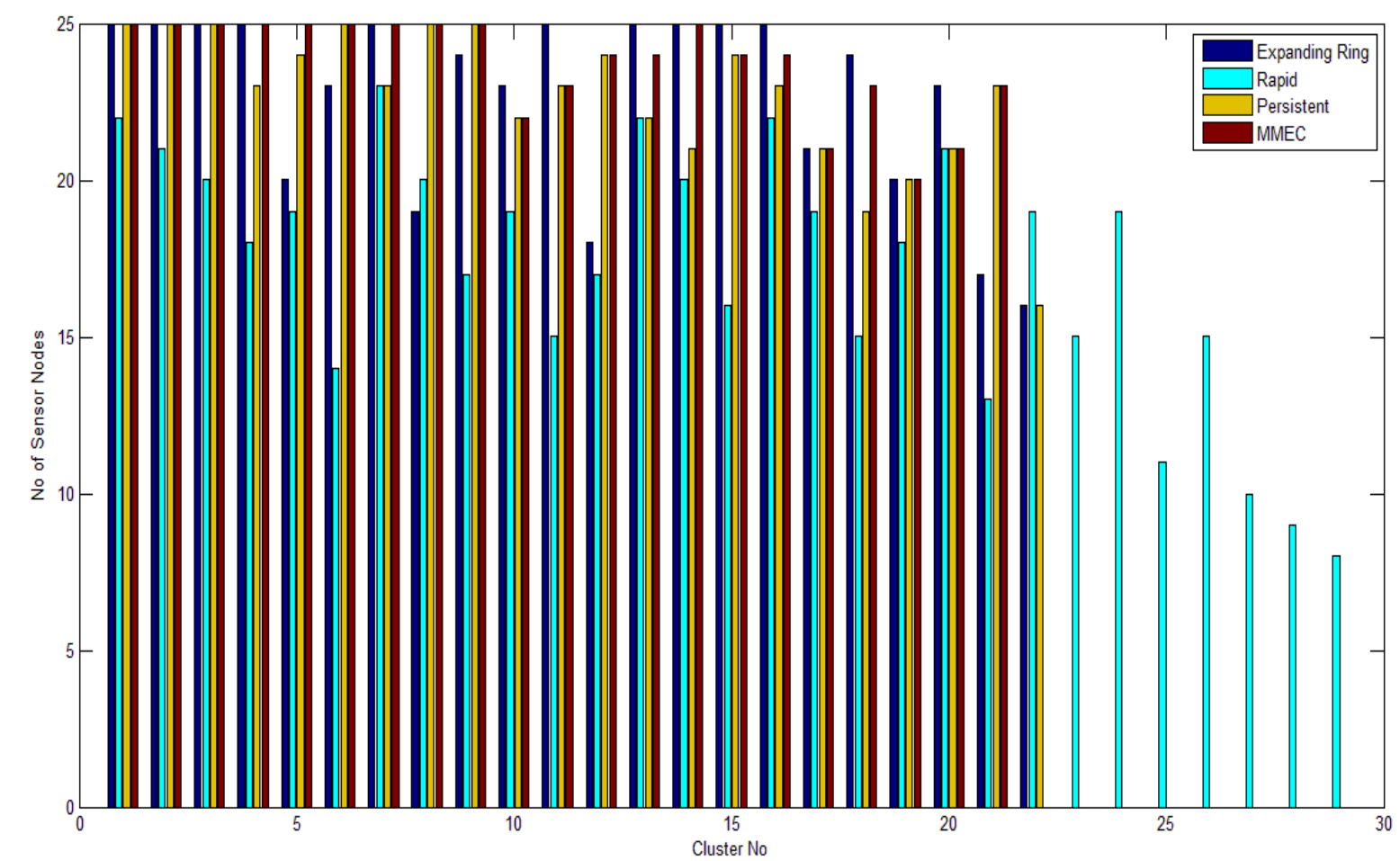

Figure 5. Size of each cluster for Expanding ring, Rapid, Persistent and MMEC clustering algorithms

In Figure 6 a comparative study of the energy spent by cluster heads for four clustering methods- LEECH, EEUC, HEED and our SMCP is presented. In Figure 7 a comparative plot of the mean residual energy (in Joule) against number of rounds for five clustering methods- LEECH, EEUC, HEED, PEGASIS and our SMCP is presented. In Figure 8 a comparative plot of nos. of alive nodes (in \%) against number of rounds for same five clustering methods is presented. This shows that our method is also efficient and better in terms of energy constraint.

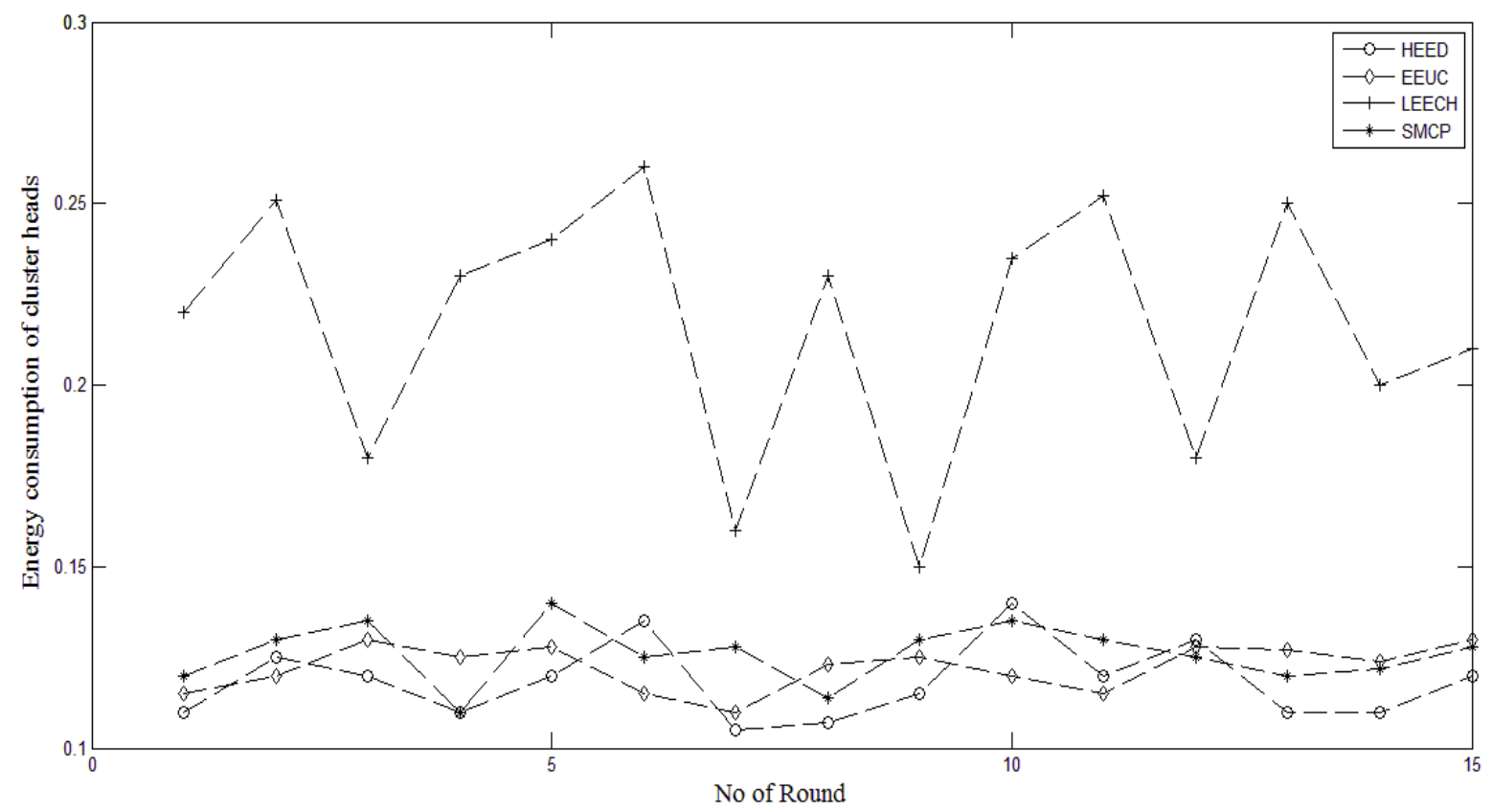

Figure 6. The amount of energy spent by cluster heads v/s Number of rounds 


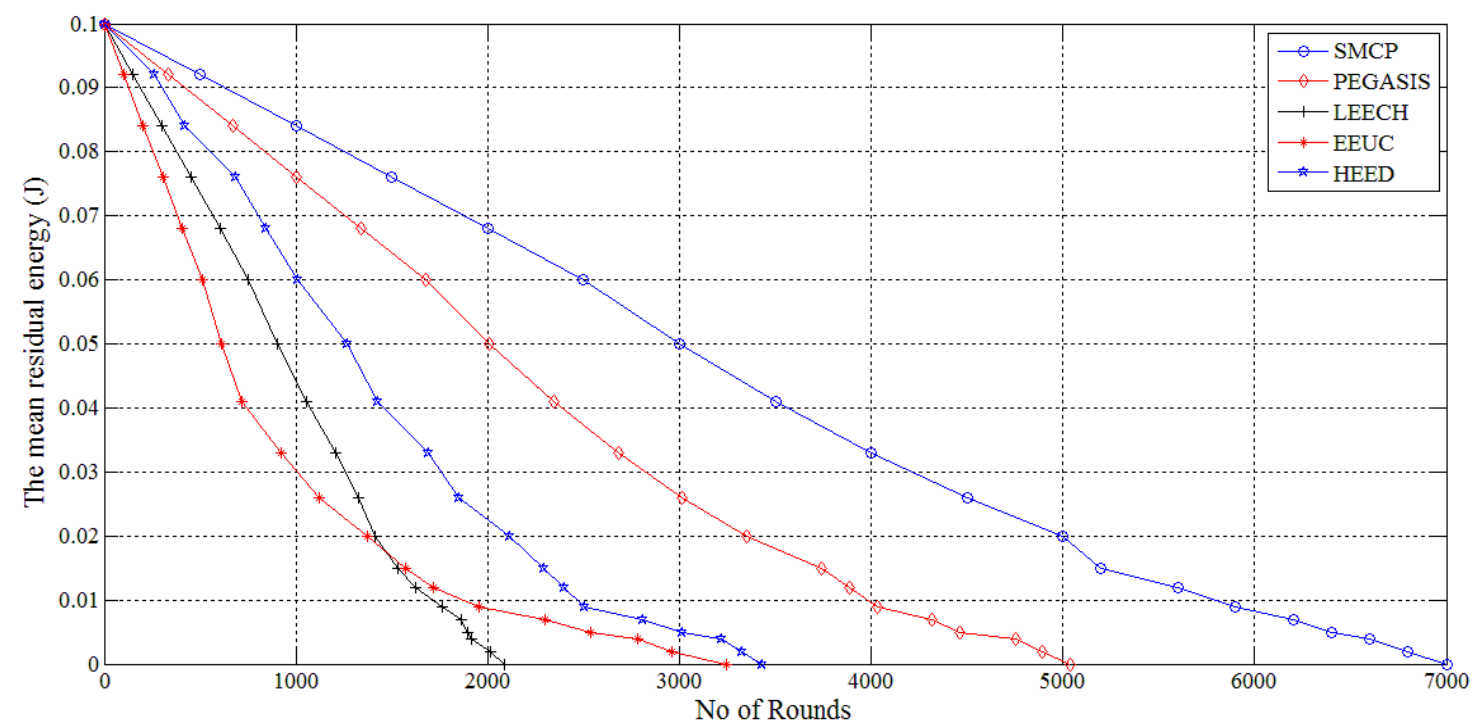

Figure 7. The mean residual energy (in Joule) v/s Number of rounds

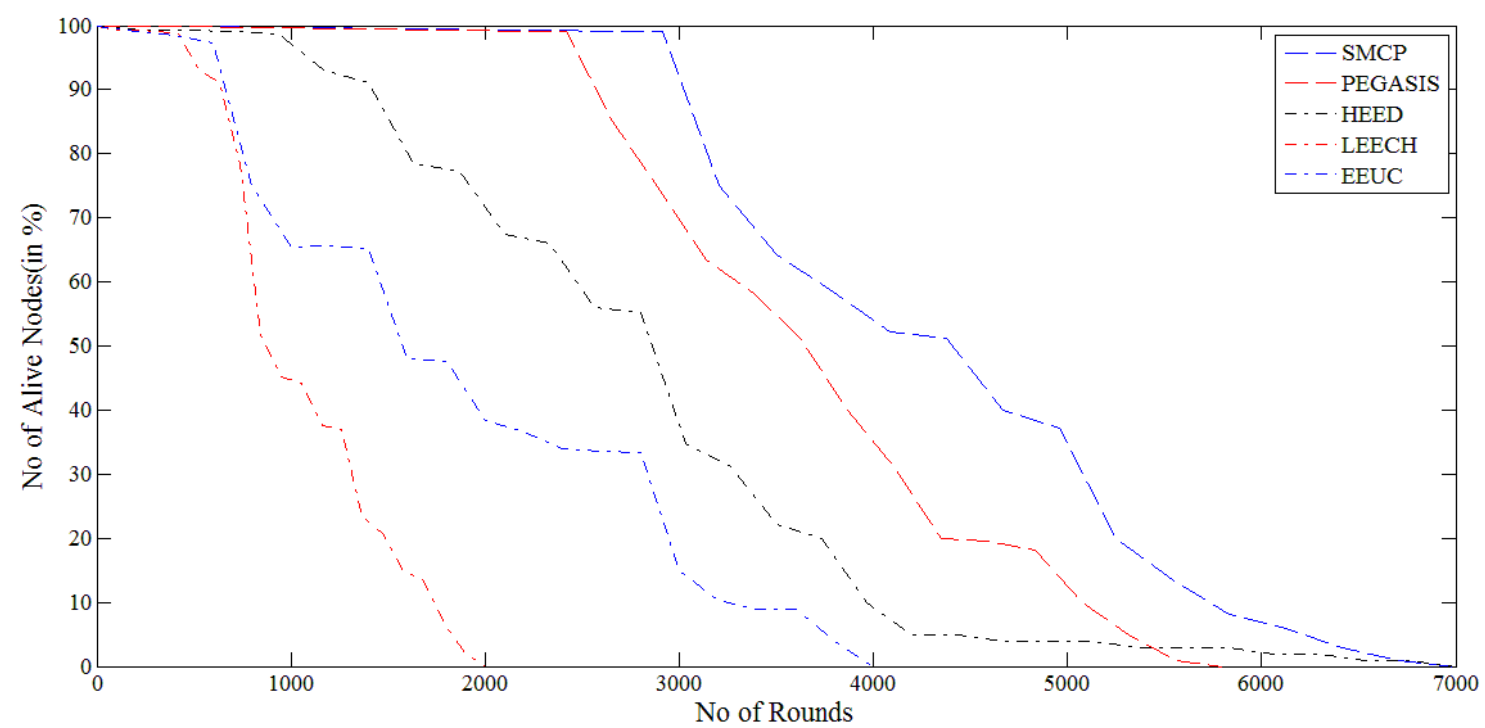

Figure 8. Nos. of alive nodes (in \%) v/s Number of rounds

\section{CONCLUSION}

We have proved that our proposed SMCP is very efficient in terms of energy awareness, message count, nos. of formed cluster etc. There are also scopes of modification in SMCP. We have implemented sequential clustering in this paper. Our further work will be focused on implementing a simultaneous clustering protocol i.e. all the cluster should form simultaneously for time saving.

\section{REFERENCES}

[1] Akyildiz, I. F., Su, W., Sankarasubramaniam, Y., and Cayirci, E., "Wireless sensor networks: a survey," in Computer Networks, Volume 38, Issue 4, Pages 393-422.

[2] Sohrabi, K., Gao, J., Ailawadhi, V., and Pottie, G.J., "Protocols for self-organization of a wireless sensor network," in IEEE Personal Communications, Volume: 7 Issue: $5,16-27$.

[3] Min, R., Bhardwaj, M., Cho, S. H., Shih, E., Sinha , A., Wang, A., and Chandrakasan , A., " Low power wireless sensor networks", in Proceedings of International Conference on VLSI Design, Bangalore, India.

[4] Rabaey, J.M., Ammer, M.J., Da Silva, J.L., Jr., Patel, D., and Roundy, S., "PicoRadio supports ad hoc ultra low power wireless networking," in IEEE Computer, Volume: 33, Issue: 7, 42-48.

[5] Katz, R.H., Kahn, J.M., and Pister, K.S.J., "Mobile networking for smart dust," in Proceedings of the 5th Annual ACM/IEEE International Conference on Mobile Computing and Networking (MobiCom_99), Seattle, WA. 
[6] Akyildiz et al. Georgia Institute of Technology. "A Survey on Sensor Networks". IEEE Comunication Magazine.

[7] Sikka, P., Corke, P., Valencia, P., Crossman, C., Swai,n D., and Bishop-Hurley, G., "Wireless adhoc sensor and actuator networks on the farm," in IPSN '06: Proceedings of the 5th International Conference on Information Processing in Sensor Networks, pages 492-499, New York, NY, USA. ACM Press.

[8] Butler, Z., Corke, P., Peterson, R., and Rus, D., "From robots to animals: Virtual fences for controlling cattle," Int. J. Rob. Res., 25(5-6):485-508.

[9] Zhang, P., Sadler, C. M., Lyon, S. A., and Martonosi, M., "Hardware design experiences in ZebraNet," in SenSys '04: Proceedings of the 2nd International Conference on Embedded Networked Sensor Systems, pages 227-238, New York, NY, USA. ACM Press.

[10] Hughes, D., Greenwood, P., Blair, G., Coulson, G., Pappenberger, F., Smith, P., and Beven, K., "An intelligent and adaptable grid-based flood monitoring and warning system," in Proceedings of the 5th UK eScience All Hands Meeting.

[11] Basha, E. A., Ravela, S., and Rus, D., "Model-Based Monitoring for Early Warning Flood Detection," SenSys '08, Raleigh, North Carolina, USA.ACM Press.

[12] Sterbenz, J.P.G., Krishnan, R., Hain, R.R., Jackson, A.W., Levin, D., Ramanathan, R., Zao, J., "Survivable mobile wireless networks: issues, challenges, and research directions," ACM Workshop on Wireless Security (WiSe), Atlanta, GA, USA, vol. 1, pp. 31-40.

[13] Yang, Y., Wu, H.H., and Chen, H.H.,"SHORT: Shortest Hop Routing Tree for Wireless Sensor Networks", IEEE ICC 2006 proceedings

[14] Lindsey, S., and Raghavendra, C.S. "PEGASIS: Power Efficient Gathering in Sensor Information Systems," in Proceedings of IEEE Aerospace Conference 2002. Big Sky, Montana. pp. 1125-1130

[15] Seetharam, A.,Bhattacharyya,A., Naskar,M..K. and Mukherjee, A.," Estimation of Node Density for an Energy Efficient Deployment Scheme in Wireless Sensor Network," In Proceedings of The 3rd International Conference on Communication System Software And Middleware COMSWARE, Bangalore, India

[16] Zheng, R., Hou, J. C., and Sha, L., "Asynchronous wakeup for ad hoc networks," in Proceedings of the 4th ACM international symposium on Mobile ad hoc networking and computing (MobiHoc).

[17] Ye, W., Heidemann, J., and Estrin, D., "An energyefficient MAC protocol for wireless sensor networks," in Proceedings of the 21 st IEEE INFOCOM.

[18] Chang, J. H., and Tassiulas, L., "Energy conserving routing in wireless ad-hoc networks," in Proceedings of the 19th IEEE INFOCOM

[19] Heinzelman, W. R., Chandrakasan, A., Balakrishnan, H., "Energy efficient Communication Protocols for Wireless Microsensor Networks," Proc. Hawaaian Int'l Conf. on Systems Science.

[20] Cadez, I. V., Ganffey, S., and Smyth, P. A, "General Probabilistic Framework for Clustering Individuals and Objects," Proceedings of the KDD, 140-149.
[21] Kleinhans, J. M.; Sigl, G.; Johannes, F. M.; Antreich, K. J. GORDIAN: VLSI Placement by Quardratic Programming and Slicing Optimization, IEEE Trans on Computer Aided Design 1991.

[22] Ramamoorthy, C.V., Bhide, A., and Srivastava, J., "Reliable Clustering techniques for large, mobile packet radio networks," in Proceedings of the 6th Annual Joint Conference of the IEEE Computer and Communications Societies(INFOCOM _87), San Francisco, CA, USA, vol. 1, pp. 218-226.

[23] Krishnan, R. and Starobinski, D., "Efficient clustering algorithms for self-organizing wireless sensor networks," Ad-Hoc Networks (4) 36-59.

[24] Joydeep Banerjee, Souvik Kumar Mitra, Pradipta Ghosh, Mrinal K. Naskar: Memory based message efficient clustering (MMEC) for enhancement of lifetime in wireless sensor networks using a node deployment protocol. ICCCS 2011: 71-76

[25] Baker, D.J., and Ephremides, A., "The architectural organization of a mobile radio network via a distributed algorithm," IEEE Transactions on Communications, COM29 (11) 1694-1701.

[26] Xu, K., Gerla, M., "A heterogeneous routing protocol based on a new stable clustering scheme," in Proceeding of IEEE Military Communications Conference, Anaheim, CA.

[27] Nagpal, R., and Coore, D., An algorithm for group formation in an amorphous computer, in: Proceedings of the 10th International Conference on Parallel and Distributed Systems ,Las Vegas, NV

[28] Baker, D.J., Ephremides, A.,and Flynn, J.A., "The design and simulation of a mobile radio network with distributed control," IEEE Journal on Selected Areas in Communications 226-237.

[29] Lin, C.R., and Gerla, M., "Adaptive clustering for mobile wireless networks," IEEE Journal on Selected Areas Communications 15 (7) 1265-1275.

[30] Ephremides, A., Wieselthier, J.E., and Baker, D.J., "A design concept for reliable mobile radio networks with frequency hopping signaling," in Proceedings of IEEE 75 (1) (1987) 56-73.

[31] Banerjee, S., and Khuller, S., "A clustering scheme for hierarchical control in multi-hop wireless networks," in Proceedings of 20th Joint Conference of the IEEE Computer and Communications Societies (INFOCOM), Anchorage, AK.

[32] Zhang, H., and Arora, A., "GS3: scalable self-configuration and self-healing in wireless networks," in Proceedings of the 21st ACM Symposium on Principles of Distributed Computing, Monterey, CA.

[33] Bandyopadhyay, S., and Coyle, E., “An energy efficient hierarchical clustering algorithm for wireless sensor networks," in Proceedings of the 22nd Annual Joint Conference of the IEEE Computer and Communications Societies, San Francisco, California.

[34] Heinzelman, W.B., Chandrakasan, A.P., and Balakrishnan, H., "Application specific protocol architecture for wireless microsensor networks," IEEE Transactions on Wireless Networking. 
[35] Demirbas, M., Arora, A., and Mittal, V., "FLOC: a fast local clustering service for wireless sensor networks," in Proceedings of Workshop on Dependability Issues in Wireless Ad Hoc Networks and Sensor Networks (DIWANS'04), Palazzo dei Congressi, Florence, Italy.

[36] Chan, H., and Perrig, A., "ACE: an emergent algorithm for highly uniform cluster formation," in Proceedings of the 1st European Workshop on Sensor Networks (EWSN), Berlin, Germany.

[37] Younis, O., and Fahmy, S., "HEED: A Hybrid, EnergyEfficient, Distributed clustering approach for Ad Hoc sensor networks," IEEE Transactions on Mobile Computing 3 (4) $366-379$.

[38] Ding, P., Holliday, J., and Celik, A., "Distributed energy efficient hierarchical clustering for wireless sensor networks," in Proceedings of the IEEE International Conference on Distributed Computing in Sensor Systems(DCOSS'05), Marina Del Rey, CA.

[39] Youssef, A, Younis, M., M. Youssef, and Agrawala, A., "Distributed formation of overlapping multi-hop clusters in wireless sensor networks," in Proceedings of the 49th Annual IEEE Global Communication Conference (Globecom’06), San Francisco, CA.

[40] Wang, K., Abu Ayyash, S., Little, T.D.C., and Basu, P., "Attributebased clustering for information dissemination in wireless sensor networks," in Proceeding of 2nd Annual IEEE Communications Society Conference on Sensor and Ad Hoc Communications and Networks (SECON'05), Santa Clara, CA.

[41] Li, C., Ye, M., Chen, G.and Wu, J., "EEUC: An energy efficient unequalclustering mechanism for wireless sensor networks," in Proceedings of IEEE MASS 2005, 8 pp. 604.

[42] Ye, M., Li, C., Chen, G., and Wu, J., "EECS: an energy efficient clustering scheme in wireless sensor networks", in: Proceedings of 24th IEEE International Performance, Computing, and Communications Conference(IPCCC 2005), Phoenix, Arizona, pp.535- 540.

[43] Yu, J., and Chong, P., "An efficient clustering scheme for large and dense mobile Ad hoc networks," Computer Communications, vol. 30, pp. 5-16.

[44] Wei, D., and Chan, H., "Clustering Ad Hoc networks: schemes and classifications," in: Proceedings of 3rd Annual IEEE Communications Society on Sensor and Ad Hoc Communications and Networks(SECON'06), Reston, VA, USA, pp. 920-926.

[45] Wang, D., "An energy-efficient clusterhead assignment scheme for hierarchical wireless sensor networks," International Journal of Wireless Information Networks, vol. 15, pp. 61-71.

[46] Yi, S., Heo, J., Cho, Y., and Hong, J., "PEACH: powerefficient and adaptive clustering hierarchy protocol for wireless sensor networks,"Computer Communications,vol. 30, pp. 2842-2852.

[47] Basagni, S., "Distributed clustering algorithm for ad hoc networks," in: Proceedings of International Symposium on Parallel architectures, algorithm, and networks (I-SPAN), Fremantle, Australia, pp. 310-315.

[48] Mallinson, M., Drane, P., and Hussain, S., "Dsicrete Radio Power Level Consumption Model in Wireless Sensor Network," Mobile Ad-Hoc and Sensor Systems', IEEE International Conference pp-1-6. 